\title{
Statistical Adjustment Module Advanced Optimizer Planner and SAP Generated the Case of a Food Production Company
}

\author{
Amelec Viloria' ${ }^{1}$ and Mercedes Gaitán-Angulo ${ }^{2^{*}}$ \\ 'Universidad de la Costa, Barranquilla, Colombia; \\ aviloria7@cuc.edu.co \\ 2Escuela de Negocios de la Fundación Universitaria Konrad Lorenz, Bogotá, Colombia; \\ mercedes.gaitana@konradlorenz.edu.co
}

\begin{abstract}
Objectives: An analysis of forecast system SAP-APO of a company producing food demand is made Methods/Statistical analysis: redefining the parameters of statistical models I module demand for SAP-APO, change the statistical model adaptive average seasonality and trend of historical data and categorize products according segmentation analysis to assign a different range of tolerance to each when comparing their demand forecasts with sales in recent months. Findings: After implementation the percentage improvement product to manually adjust decreased by $12.58 \%$, compared to the previous level of attention. Application/Improvements: This decreased attention, resulting in an increase in the utility system more than $85 \%$.
\end{abstract}

Keywords: Information Systems, Inventory Management, SAP Advanced Planner \& Optimizer Module,

\section{Introduction}

Currently organizations require an information infrastructure that allows them to make accurate decisions based on real - time data and market intelligence elements. New developments in technology improvements in planning the supply chain are becoming more sophisticated and have become gradually key performance differentiators.

One of the most efficient and important today, technological developments is generated by SAP APO module, which has many features specifically designed to support companies in the process of Planning Supply Chain. The Demand Planning is one of its features, which is responsible for generating accurate forecasts and production plans by analyzing the historical planning and business intelligence ${ }^{1}$.

The company under study is one of the leading producers and distributors of food in Latin America; this has offices in Venezuela and Colombia. Currently, statistical forecasts generated by its system optimization and advanced planning (SAP-APO, for its acronym in English); they are not adjusted to the volumes needed by the business. The statistical model that chooses the system is not the most appropriate for each product as forecasts show very high deviations or equal proposals for each month, so it must be adjusted manually. 
The research is to propose improvements to the SAPAPO system through analysis and adjustment of their statistical models and parameters to the behavior of sales for the following categories of products that makes up the company's sales portfolio: ABA (Animal Feed) oils, Rice, Tuna, corn Flakes, Detergents, Corn flour, Ice Cream, Soap, ketchup, Margarine, Pets, Mayonnaise, Modifiers dairy and pasta. It is expected that the above increase efficiency thereof.

\section{Groundwork}

Today businesses depend largely on your environment, which, is influenced by events or situations of nature: cultural, technological, political, economic, ecological, demographic, etc. or combination of these ones and interactions.

Similarly, global competition, volatile demand, the specific needs of customers and cycles ever shorter product life, along with other factors determine profound changes in the way of conducting relations between manufacturers, suppliers, distributors, customers and consumers 2 .

Business success depends on continuous and systematizes co process of adaptation and adjustment to their environment and finding a strategic position in the market to ensure the profitability of its operations.

In this vein, companies should focus their abilities and skills to properly manage their resources and ensure efficient supply chain and thus meet the needs of its customers ${ }^{3}$.

Such satisfaction is not only based exclusive you in product quality; so are "the when" and "where" it is essential to ensure product availability when and where the customer requires.

The use of a technology platform that allows them to make accurate decisions in real time and with elements of business intelligence specializing in the optimization of the Planning supply chain is essential ${ }^{4}$.

In order to improve the management of the supply chain the internship project, conducted in a beverage company consumer, which showed the need to maintain and ensure the balance of returnable materials within the chain emerges supplies of the organization.

The project is to design and implement a model processes to estimate the recovery of returnable materials market using the advanced planning SAP Advanced
Planner \& Optimizer (APO), and thereby estimate a procurement plan associated with the existence inventory and recovery ${ }^{5}$.

This project is part of an initiative that includes the implementation of the aforementioned tool, to support all processes PLANNING ng supply a major beverage company.

\section{Methodology}

The methodology is presented in which the steps up each of the phases were clarified ${ }^{6,7,8}$ :

- Phase 1. Define: Business induction interaction with tools and systems generation process statistical forecasting.

- Phase 2. Measure: choice of sample size to study, obtaining previous forecasts, Stock Keeping Unit (SKU) classification, story time and life cycle, obtaining average sales last month, description of statistical models and parameters.

- Phase 3. Analyze and diagnose phase: analysis of the current situation through the signal indicator tracking, identification and causes of restrictions, Strengths, Weaknesses, Opportunities and Threats (SWOT) analysis forecasts generation system.

- Phase 4. Plan and execute: setting and defining the parameters of the models proposed exchange of statistical models, categorizing SKU for review of forecasts, and analysis results implemented and establishment of improvements made in the proposed process.

- Phase 5. Control: generation of indicators.

\section{Results and Discussion}

The results of each phase are described below.

\subsection{Phase I: Definition}

Four (4) basic steps for the generation of statistical forecasting demand established in the company study, using historical values and statistical forecasting methods, which are described below:

\subsubsection{Collection of Historical Data}

This activity is loaded into the information system each of the products in the portfolio. It reviews and adjusts the horizon of history to use (number of months in the past) 
for calculating the statistical forecasts. This is done on the first day of each month for the following month forecasts onwards and takes day and a half to run.

\subsubsection{Validation of History}

History settings are enabled to use (number of months) for calculating the statistical forecasts. If inconsistencies in the story (months with zero sales figures) are observed is channeled to the department. This activity is performed at the end of the data collection it must be concluded not later than the second day of the current month.

\subsubsection{Forecast Calculation}

Calculating corrected orders for previous months, that is, orders for the month less unsatisfied demand the previous month running. Then automatically segmenting products for the forecast in the corresponding string to run on three, six or twelve months (time to make history for each product). To finally run the chain statistical forecasting to twelve (12) months, which includes statistical models and leads to the final results through the choice of model to shed less error? This activity is carried out in half a day of work after the validation of the story is completed?

\subsubsection{Revision Forecast}

It analyzes of cases where the statistical model automatic selection is not the best, based on comparing the generated forecast for the following course month with the average sales of the last three (3) months each product, establishing a tolerance of $5 \%$.

\subsection{Phase II: Information Collection}

Foods that distribute the company under study are divided into fifteen (15) categories and a total of three hundred (300) products. In this project we study only one group of these, establishing their choice in the calculation of sample size for a finite population of 108 products taking into account the SKU for the categories of flour, detergents and soaps.

\subsection{Phase III: Analysis and Diagnosis}

After selecting the products to be studied, these forecasts were collected, thrown in the month of July 2012 and containing forecasts for the next twelve (12) months. Looking at the data generated by the system, the products are divided into three (3) large groups, these are:
Constants forecasts: products that present the same proposals for the next twelve (12) months.

Forecasts outside the tolerance range: products presenting proposals when compared with its average sales of the last three (3) months, differ by more than $5 \%$.

Forecasts that need to be adjusted manually: products that have constant forecasts, outside the range of tolerance or both.

Of the one hundred and eight (108) products taken from sample, fifty-nine (59) of these yielded constant forecasts, which represents $54.63 \%$ of the total. Moreover, eighty-two (82) products present forecasts that are outside the tolerance range established, that is, the $75.93 \%$ of the sample. This leads to the $87.96 \%$ of the products need to be adjusted manually, i.e., ninety-five (95).

The models used by the SAP-APO system are:

- Model 1: adaptation constant alpha

- Model 2: moving average

- Model 3: moving average pond erated

- Model 4: test of seasonality

- Model 5: adaptive forecast

- Generic model: regression linear

\subsection{Phase IV: Proposed Improvements and Results Implementation}

As for the model 1, the variation was made in the alpha parameter, leaving its forecast period of twenty - four (24) months as was previously but failing to take into account only six (6) of the last months of history (offset).

In model 2, the forecast period remained the same but the offset was modified in order to take closest to the current date to describe the behavior coming presenting the products of the sample values since one of the characteristics of the model is which is not easy to determine a forecast period according to the data, therefore remained interim periods if the model is not quickly adapted to changing trends or seasonality.

In the case of model 3 it was modified to offset not varied considerably from the current date as the alpha decreases exponentially parameter, whose values were left in the same interval as they were configured ( 0.05 to 0.90).

The model 4 acts with an exponential smoothing, so as model 3 assigns greater weight to recent data history according to the alpha range (0.05 to 0.090), in this case the forecast period was extended but the offset is 
decreased because if is much fluctuation with respect to recent data will shed many mistakes. Model 5 for adjusting the model 3 as because they have the same strategy forecast is the weighted moving average was performed.

For the generic model, which represents a linear regression model alpha value it was considered appropriate because it was quite high but the beta and gamma values increased to 0.5 because this way could soften the lines of trend and seasonality of those products that required it. Overall the sigma parameter was increased for all models to 1.5 as by default the system assigned the value of 1.25 and as this corrects the historical values that are far from the forecast, the greater is its value, the prognosis resemblance to actual history.

After defining the new parameters of each model, and replaced the model of adaptive average for test of trend and seasonality, they run to obtain forecasts for the next twelve (12) months, were made from the month of August 2012, where improvements were observed, which resulted in more accurate and reliable forecasts. Also forecasts were evaluated as performed previously, noting that these were constant percentage and which left the margin of error established from the outset (5\%).

By obtaining these statistics the percentage decrease in each group note regarding the run from the previous forecast month in July 2012 to give a percentage of $5.56 \%$ constant forecasts which represent only 6 products sample. In the case of those forecasts outside the range of tolerance of $5 \%$ the figure fell to 23 products total, which involves $21.30 \%$ of the sample to reach a total of 27 products that need adjustment, i.e. 25.01\%.

\subsection{Phase V: Control}

Finally, two (2) indicators for monitoring and analysis so as to bring direct control and continuous planning process were generated demand, and forecasts proposals generated by the system.

Tracking signal indicator: corresponds to an accuracy indicator for evaluating the accuracy of the forecasts generated by the SAP-APO system. The main objective of this indicator is to assess whether the process is carried out is under control, a time considered normal parameters defined. Since the profile of demand forecasting has different parameters that influence the data produced, review each case why after obtaining the demand forecast. On the other hand, this indicator can also be assessed globally, in which the level of care they need statistical models found in the system will be reviewed.
Alert Indicator: with the premise that forecasts thrown by the system are the final proposals that will define the dynamic plan Business Alimentos Polar, the second indicator is framed in the percentage of care that they should be given the forecasts based on the usual calculations of differences with actual sales. In this sense, the main objective is the assessment of forecasts and the system requires much attention, contributing to the profitability of the business. The desired this indicator level is $0 \%$, because if the system is capable of generating effective proposals, Analysts should not have the need to modify these system - generated forecasts, in this sense, the goal is for the system to generate distribution proposals adjusted to reality and what $s$ requirements Planning Analyst.

\section{Conclusion}

Proposals for improvements were reviewed by the "management demand planning and master data" and then approved, he began the implementation process. Once implemented, ongoing monitoring of the results of the proposed demand generated by the system and the percentage of predictions that did not meet previously established requirements is performed. It is noted that thanks to the changes made to the module DP "Demand Planning", the percentage of products to be manually adjusted decreased by $12.58 \%$ compared to the previous level of attention. This decreased attention, resulting in an increase in the utility system more than $85 \%$.

\section{References}

1. Stang D, Arcuri G. SAP my SAP advanced planner \& otpimizer Supply Chain Planning (SCP) applications. Gartner Product Report; 2002.

2. Peterson K. SCM solutions multienterprise will come of age by 2012. Gartner Research Note; 2013.

3. Davies et al. How to get the MOST out of your supply chain ?- An overview of APS systems in the consumer products manufacturing and process industry. Report of Deloitte Consulting; 2002.

4. Walravens P, Shu M. Understanding supply chain management software. Lehman; 2011.

5. Stang D, Arcuri G. SAP my SAP advanced planner and otpimizer Supply Chain Planning (SCP) applications. Gartner Product Report; 2012. 
6. Stadtler H, Kilger C. Adn supply management advanced planning. Ed Springer; 2013.

7. Amelec V. Methodology to increase the adaptability and flexibility of the supply chain of automotive company through lean manufacturing. Advanced Science Letters. 2015; 21(5):1517-20.
8. Amelec V, Carmen V. Design of a model of evaluation of productivity for microfinance institutions. Advanced Science Letters. 2015; 21(5):1529-33.

9. Amelec V, Carmen V. Validation of a model for productivity evaluation for microfinance institutions. Advanced Science Letters. 2015; 21(5):1610-4. 